EUROPEAN ORGANIZATION FOR NUCLEAR RESEARCH

CERN — AB DEPARTMENT

CERN-AB-2005-057

CLIC Note 640

\title{
CLIC 50 MW L-Band Multi-Beam Klystron
}

\author{
E. Jensen and I. Syratchev
}

\begin{abstract}
$50 \mathrm{MW}$ power sources at $937 \mathrm{MHz}$ will be needed to accelerate the CLIC drive beams. We present a novel MBK concept with a large number of beamlets; this allows for small single beam perveance and high efficiency. The MBK features disc-shaped RF circuits operated in a whispering-gallery mode - a configuration permitting both high interaction impedance and easy spurious mode damping.
\end{abstract}

Submitted to the 7th International High Energy Density and High Power RF Workshop, Kalamata, Greece, 13-17th June 2005 


\title{
CLIC 50 MW L-Band Multi-Beam Klystron
}

\author{
Erk Jensen and Igor Syratchev
}

CERN, 1211 Geneva 23, Switzerland

\begin{abstract}
MW}$ power sources at $937 \mathrm{MHz}$ will be needed to accelerate the CLIC drive beams. We present a novel MBK concept with a large number of beamlets; this allows for small single beam perveance and high efficiency. The MBK features disc-shaped RF circuits operated in a whispering-gallery mode - a configuration permitting both high interaction impedance and easy spurious mode damping.
\end{abstract}

Keywords: Multi-Beam Klystron, MBK.

\section{INTRODUCTION}

The CLIC (Compact Linear Collider) study for an electron-positron in the multi$\mathrm{TeV}$ range is based on a two-beam scheme [1]. The CLIC study team is proposing high-power $(\sim 50 \mathrm{MW})$, long-pulse $(\sim 100 \mu \mathrm{s}) 937 \mathrm{MHz}$ multi-beam klystrons to supply the RF power needed to accelerate the low-energy, high-intensity drive beam. These klystrons should meet the minimum performance specification [2], given in Table 1. A multi-beam klystron (MBK) has been specified because it is believed that this is the only device that can achieve high efficiencies at these high powers.

\begin{tabular}{|c|c|c|}
\hline Parameter & Value & Unit \\
\hline RF pulse width & 100 & $\mu \mathrm{s}$ \\
\hline Pulse repetition frequency & 100 & $\mathrm{~Hz}$ \\
\hline Klystron peak RF power & 50 & MW \\
\hline Klystron average power & 500 & $\mathrm{~kW}$ \\
\hline Efficiency & $\geq 65$ & $\%$ \\
\hline Gain at saturation & $\geq 43$ & $\mathrm{~dB}$ \\
\hline Beam voltage & $<220$ & $\mathrm{kV}$ \\
\hline
\end{tabular}

Multi-beam klystrons have been developed in Russia and America in the mid-1960's [3]. An MBK is basically a parallel assembly of many low-current klystrons, which use common RF structures for the interactions with the beams. Having a large number of beamlets in the klystron enables the power per beamlet to be considerably reduced leading to lower current densities and a very low perveance per beam. Beam perveance, defined as the current per beam divided by the $3 / 2$ power of the voltage $\left(I_{B} / \sqrt{V^{3}}\right)$, is a very important parameter which determines to a large extent the power conversion efficiency (see below).

Unfortunately multi-beam klystrons that meet the above specification are not currently available, and further design and development work will be needed to demonstrate that this specification can be achieved. This paper summarizes the 
features of a new MBK design concept. It is based on results and experience with the Barrel-Open-Cavity (BOC) technology, which was initially developed in Russia [4], but has been further developed at CERN [5]. This new concept may lead to a mechanically robust MBK design with a potential to obtain an efficiency of $80 \%$.

\section{EFFICIENCY DEPENDENCE ON PERVEANCE}

Perveance characterizes the dependence of the current on the anode voltage in the regime of space-charge limited (Child-Langmuir) emission, and it is approximately proportional to the (single) cathode area divided by the cathode-to-anode spacing squared. Optimization of the power conversion efficiency of a klystron ( $\eta=\mathrm{RF}$ output power/DC beam input power) is closely linked [6] to drift length, beam radius and perveance as well as RF cavity tuning. The RF output current (and power), due to the input modulating voltage, is a maximum when the klystron transconductance $g$ is a maximum $\left(g=I_{\text {out }} / V_{\text {in }} \propto b^{\prime} / \sqrt{K}\right.$ where $b^{\prime}$ is the normalized beam radius and $K$ the perveance). It has been shown [7] that $g$ is maximum for an optimized beam radius when the perveance is at a minimum. High efficiency klystrons $(>75 \%)$ therefore require low perveance.

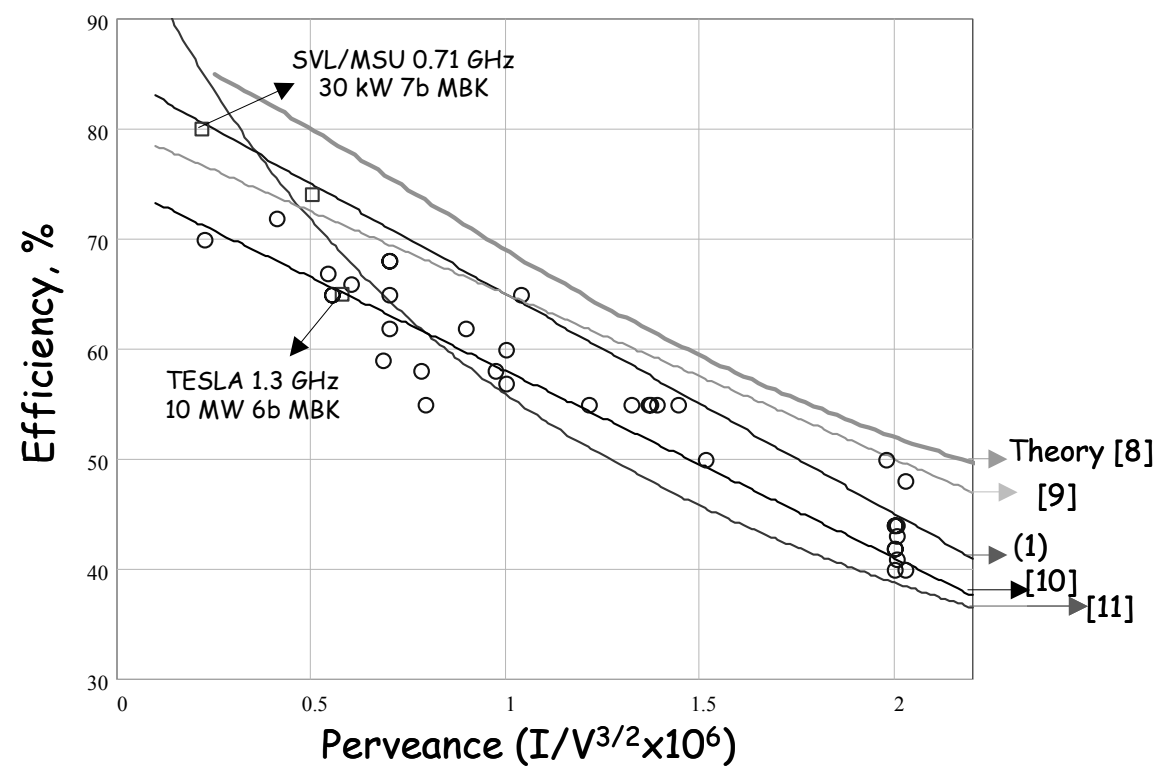

FIGURE 1: State-of-the-art single (circles) and multi-beam (rectangles) klystron efficiencies versus single beam perveance.

Since there is an upper technical limit to the voltage that can be applied, low perveance can only be obtained by operating with low currents, and this requirement, for single beam klystrons, is inconsistent with the need for high power. The idea of the $\mathrm{MBK}$ is to operate many low-current (low perveance) klystrons in parallel (using common RF structures) to generate high power at high efficiency.

Figure 1 gives efficiency-perveance data for a selection of state-of-the-art klystrons for different frequencies and power levels. Many different fits for such data can be 
found in the literature [8-11], but the low-frequency, high-efficiency MBK data [12] is best fitted by the empirical law:

$$
\eta=0.85-0.2 \cdot \mu K
$$

This fit indicates that for very-low-perveance devices, efficiencies in excess of $80 \%$ are within reach. A similar conclusion was reached by Farkas [13], who predicted efficiencies of $90 \%$ for the case of a gridded-gap, non-relativistic klystron with no space-charge effects. It therefore seems reasonable to assume that such high efficiencies are indeed attainable for a low-frequency MBK with a large number of beamlets and hence a lower current per beam and a lower cathode voltage. Based on (1), the klystron output RF power for an MBK can be expressed as:

$$
P=\eta I_{b} N_{b} V_{b}=\left(0.85-0.2 \cdot \frac{I_{b} \cdot 10^{6}}{V_{b}^{3 / 2}}\right) \cdot I_{b} N_{b} V_{b},
$$

where $I_{b}$ is the single beam current in amperes, $V_{b}$ is the beam voltage in volts, and $N_{b}$ is the total number of beamlets. Figure 2 gives the efficiency and single beam current versus number of beamlets for different cathode voltages for an output RF power of $50 \mathrm{MW}$.
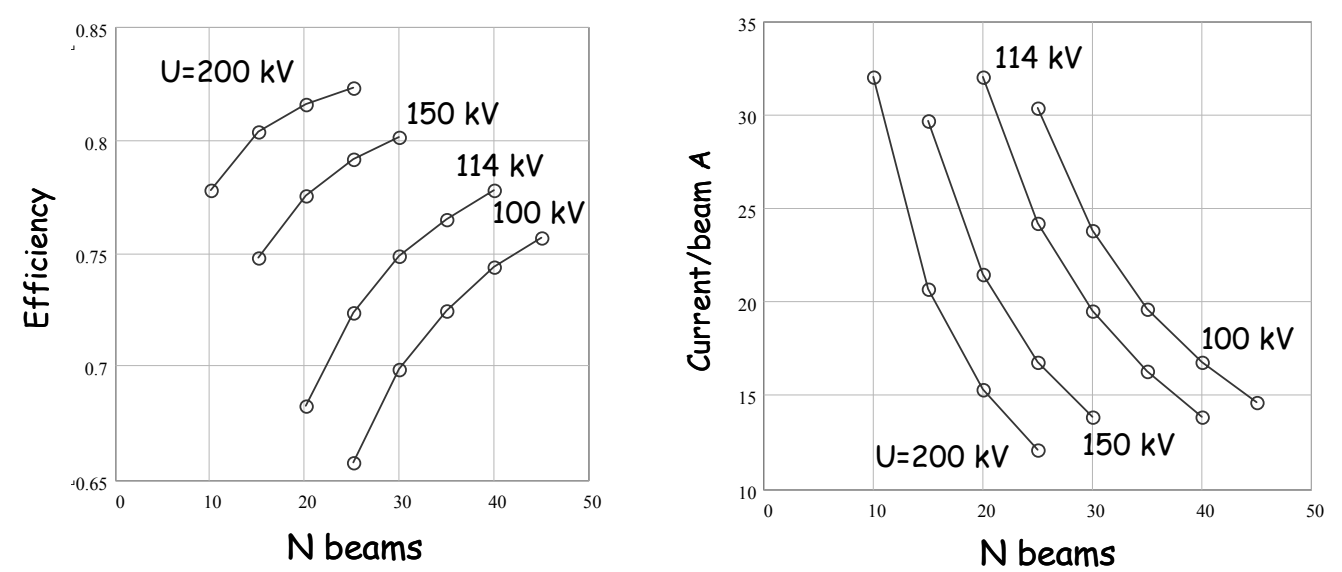

FIGURE 2: Efficiency (left) and MBK's single beam current estimates (right) versus total number of beamlets for different cathode voltages.

If we target an efficiency of $\sim 80 \%$, a beam voltage $V_{b}=150 \mathrm{kV}$ seems reasonable for the design of the klystron modulator. Choosing 30 beamlets results in a single beam current of about $15 \mathrm{~A}$.

\section{MBK RF CIRCUIT - THE DDC}

We conclude from the above that we need a large number of beamlets. However, the number of beamlets is limited by geometric, RF and beam quality considerations: These include the minimal distance between single beamlets coming from demands on cathode loading, the use of second harmonic cavities, space charge effects, the danger of exciting spurious mode instabilities, and the overall size of RF cavities. In the following, we will compare our novel approach to various other designs that have 
been proposed to solve these problems. We limit this discussion to MBKs with common RF cavities; cluster klystrons [9] and sheet beam klystrons [14] are not considered.

We will look at four different cases of field distributions in RF cavities that may be considered for use in an MBK ( $c f$. Fig. 3). Cases 1 and 4 depict modes in a disc shape (radial) cavity, while cases 2 and 3 represent modes in an annular (waveguide type) cavity. These modes have very different $R / Q$ values $(R / Q$ gives the square of the voltage as seen by the beam for a given stored energy in the cavity). Figure 4 shows the radial distribution of the axial electric field for these cases.
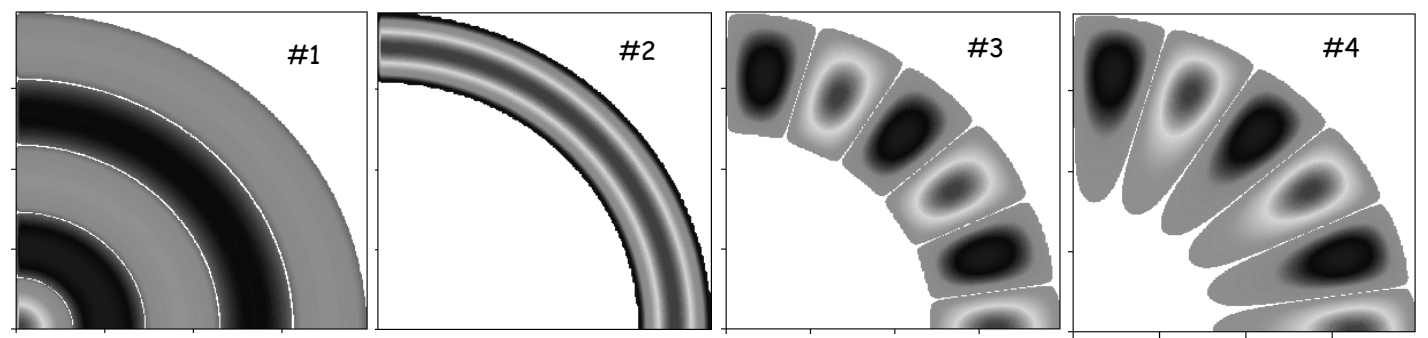

FIGURE 3: Different possible field distributions for MBK cavities (cases 1-4 from left to right).

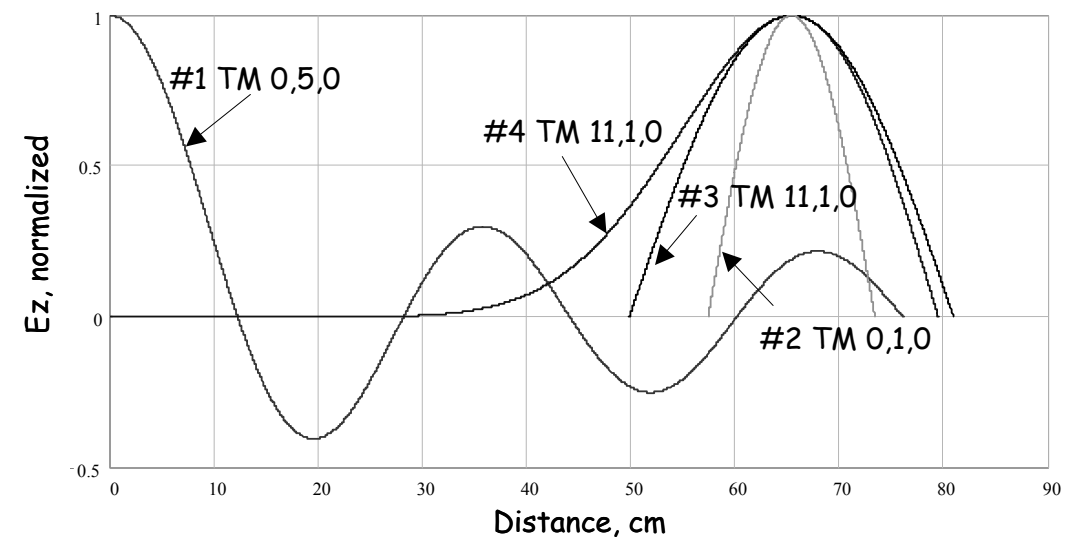

FIGURE 4: Radial distribution of the axial electric field for the different cases shown in Fig. 3.

The conventional MBK operates in a simple $\mathrm{TM}_{0,1,0}$ (pillbox type) cavity. The beamlets (typically between 6 and 30) pass through the cavity at different angular (and possibly also different radial) positions, close to the central maximum of the axial electric field. With these devices, very high efficiencies $(80 \%)$ have been demonstrated [12], but they have been achieved for relatively low RF power levels (tens of kilowatts). Obtaining higher power from this device would require an increase in the beam current, which for this geometry is soon limited by both space-charge effects and cathode loading. One way around this limitation is to change the operating mode of the cavity to a higher radial index mode (case 1). The TESLA $1.3 \mathrm{GHz}$, $10 \mathrm{MW}$, 6-beam MBK for example, uses the $\mathrm{TM}_{0,2,0}$ mode [15] (cf. Fig. 3, \#1). Another possibility was studied at SLAC for a $1.5 \mathrm{GHz}, 2 \mathrm{GW}, 10$-beam MBK [16]: the design was based on the lowest mode of an annular cavity ( $c f$. Fig. 3, \#2). The same scheme was also studied, but eventually abandoned, by Calabazas Creek 
Research for their $50 \mathrm{MW}, \mathrm{X}$-band, 8-beam MBK [17]. Finally a $150 \mathrm{MW}, \mathrm{X}-$ band, 6beam MBK was proposed by KEK [18], based on a waveguide ring cavity ( $c f$. Fig. 3, \#3). The CLIC $50 \mathrm{MW}, 937 \mathrm{MHz}$ design is based on a $\mathrm{TM}_{m, 1,0}$ mode of high azimuthal order $m$ ("whispering gallery" mode) in a disc-shaped cavity and has 27 beams (cf. Fig. 3, \#4). In this device the total number of beamlets is equal to about twice the index $m$ of the operating mode. If we try to design a 27-beam MBK using the TESLA approach, we would have to work with the $\mathrm{TM}_{0,5,0}$ mode. A major drawback here would be the drastic drop of $R / Q$ due to the energy stored in that part of the cavity that does not interact with the beam. To keep $R / Q$ high, we need the energy stored only around the beam interaction area, as for example in cases 2 to 4 . For a given gap length and voltage, the impedance ratio is simply the inverse ratio of stored energies normalized to the individual $Q$-factor of each. Whispering gallery modes are thus clearly advantageous to higher order $\mathrm{TM}_{0, n, 0}$ modes.

Another very important issue for an MBK design is the parasitic mode spectrum. Strong damping of parasitic modes is absolutely necessary; otherwise they lead to unwanted instabilities and spurious oscillations, particularly dangerous at high power operation. It is clear that this problem will be particularly serious for case 1 where it is difficult to apply any damping, and for case 2 because the operating mode is at the cutoff frequency of the waveguide, resulting in a very dense mode spectrum. Looking at the remaining cases 3 and 4, we state that the disc cavity has a denser mode spectrum to start with. However, there is a unique and natural way to damp unwanted modes in the disc cavity, which seems inaccessible to the ring cavity, since it uses a unique feature of the whispering gallery modes:

It is a well-established fact that in the Barrel-Open-Cavity (BOC), which is used for high frequency RF pulse compressors [4, 19], the mode spectrum can easily be made extremely sparse. Only the whispering gallery modes have their electric and magnetic fields concentrated near the outer rim - all other modes have non-vanishing fields in other areas of the cavity. When the cavity is open, these modes radiate their energy, thus reducing their $Q$, leaving only the whispering gallery modes unaffected - see for example the measured X-band BOC spectra [19] for closed and open faces shown in Fig. 5.

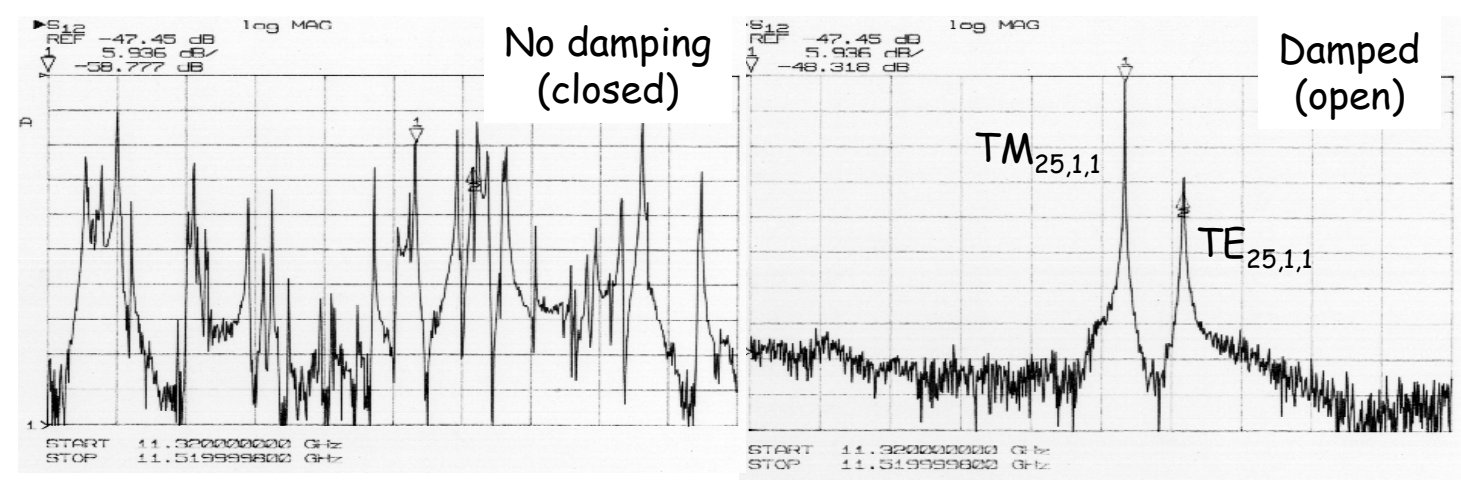

FIGURE 5: Measured spectra of an X-band BOC cavity.

For the disc cavity, the "open" conditions may be replaced by an RF absorber, which can be conveniently placed inside at a radius where the operating mode is not 
affected. Experience gained with the $3 \mathrm{GHz}$ BOC RF pulse compressor for the CLIC Test Facility 3 (CTF3) [20] validates this approach.

Simulations of a damped disc cavity (DDC) geometry have been made using HFSS. A silicon carbide ( $\mathrm{SiC}$ ) absorber ring was placed at a position which produced a $10 \%$ reduction of the operating mode $Q$-factor from $3.3 \times 10^{4}$ to $3.0 \times 10^{4}$. The $Q$-factors of all modes with a radial index of three or higher were reduced by more than a factor of 1000; we assume that a $Q$-factor reduction of about 100 is sufficient to eliminate any effects on the beam.

A general view of the DDC is shown in Fig. 6. The beam pipe diameter is rather small ( $\sim 1 / 16$ of the operating wavelength) and takes advantage of the low singlebeam current and low frequency (see Table 2 below). As a result the fringe fields decay very rapidly resulting in an ideal electric field distribution; the electric field remains very constant, within $0.1 \%$, across the beam waist $(\lambda / 32)$.

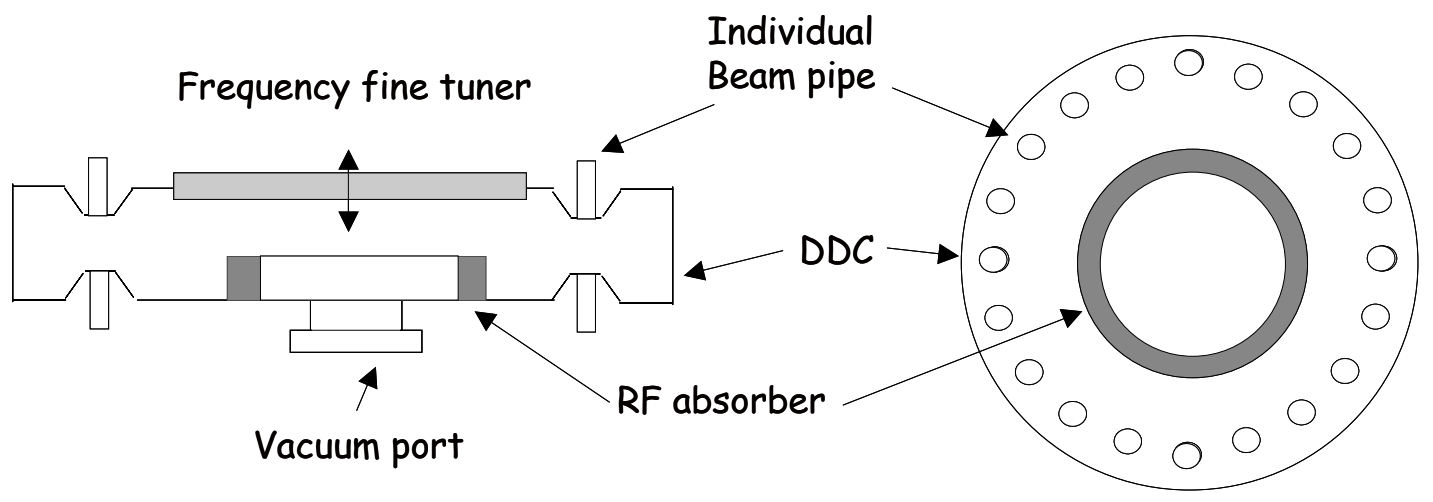

FIGURE 6: General view of the DDC.

The inter-beam space charge effects are minimized by the fact that the transverse beam spacing in a cavity is about half a wavelength $(\sim 16 \mathrm{~cm})$. A good vacuum is necessary $\left(10^{-8}\right.$ mbar or better) to avoid beam instabilities due to the ionization of residual gas molecules [16]. The DDC, unlike the other geometries, can be very effectively pumped by mounting a high vacuum conductivity port (diameter $\sim 40 \mathrm{~cm}$ ) on the central part of the cavity as is shown in Fig. 6. For the gain cavities, the cavity impedance can be adjusted to the required value by a careful dimensioning of the RF absorber. The fine-tuning of the cavities can be accomplished as indicated in Fig. 6.

Like for the BOC, input and output coupling is implemented using a rectangular waveguide clasped around the entire cavity, its width chosen such that the phase velocities of the waves in both the waveguide and the cavity are identical at the operating frequency. Coupling to the cavity is made through many small coupling holes in the wall between the cavity and the waveguide. The best matching to the cavity is obtained when the distance between coupling holes is half the operating mode wavelength - the total number of holes is therefore $2 \times m$. The electric field plot in the cavity, simulated with HFSS, is shown in Fig. 7 (cf. also Fig. 8). Such a configuration provides good matching to the cavity and reduces the probability of breakdown - the $3 \mathrm{GHz}$ BOC cavity was processed up to $80 \mathrm{MW}$ without any serious breakdown problems [20]. 


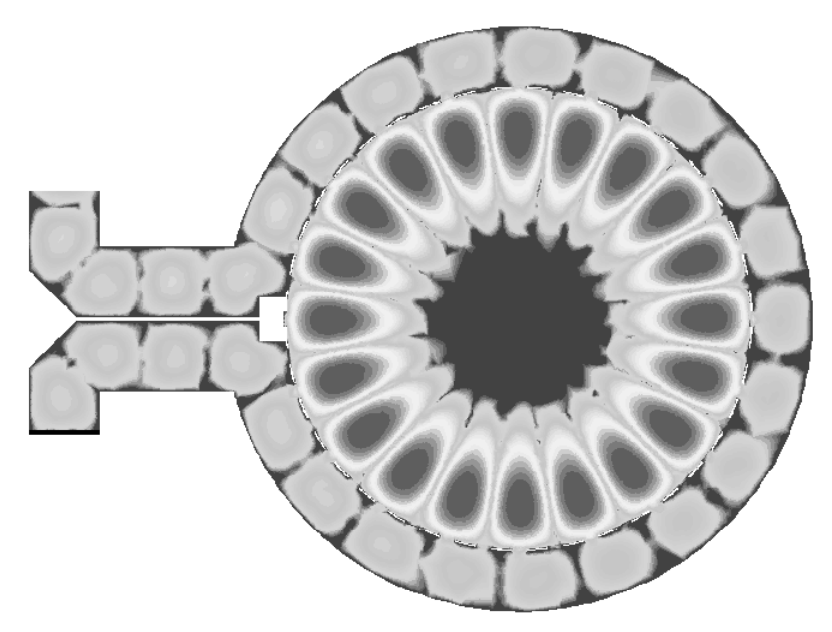

FIGURE 7: Electric field plot in a BOC median plane.

\section{MULTI-MEGAWATT MINI-WINDOWS CONCEPT}

Klystron ceramic output windows are probably the most delicate RF components in the whole system. There have been a few recent innovations and developments in this area, such as the $\mathrm{H}_{01}$-window [17, 21], mixed-mode travelling-wave window [22], and others. Scaling these solutions to our low frequency application results in extremely large windows, which would be difficult to manufacture and difficult to cool during operation. Our novel approach, the concept of which is shown in Fig. 8, makes use of the distributed coupling to the DDC operating mode described above.

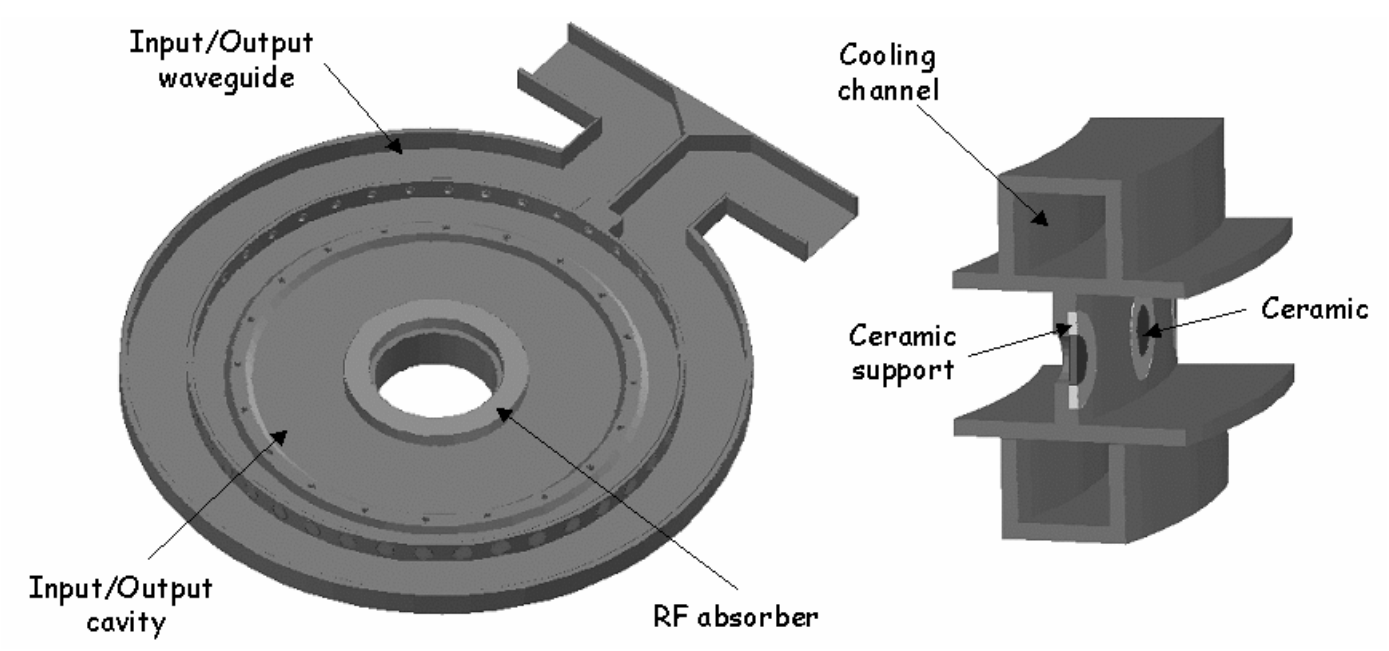

FIGURE 8: Conceptual view of the output cavity with incorporated mini-windows.

The "window" is in fact a series of many mini-windows, each covering an individual coupling hole as shown in Fig. 8. The single mini-window is first brazed into its own support and then is electron-beam welded, or even clamped, into the inner 
wall of the waveguide. Typical dimensions of the ceramic disc are $2 \mathrm{~mm}$ thickness and about $30 \mathrm{~mm}$ diameter. The DDC field configuration is such that there is no notable electric field at the mini-window location. With $2 \times m$ mini-windows, the local power flow density will be drastically reduced. For example with $m=11$ and $P=50 \mathrm{MW}$, only 2.3 MW will be transferred through each window. As a result, high reliability can be expected.

\section{CATHODE SPECIFICATION}

The current density is the parameter that defines the cathode configuration. It is exponentially proportional to the temperature, and also inversely proportional to the exponential of the work function of the cathode material (Richardson-Dushman equation). To keep beam compression as low as possible we would like to increase cathode loading, but this would increase the surface temperature and reduce the lifetime. Oxides of alkaline earth metals such as barium and strontium are added to the tungsten cathode to reduce operating temperatures. The lifetime of a klystron is primarily determined by cathode end-of-life emission, a result of barium depletion at the cathode surface. Choosing a cathode current density of $2 \mathrm{~A} / \mathrm{cm}^{2}$ (the same as the TESLA MBK proposal [15]) would allow a lifetime of 145,000 hours.

The parameters that define the gun-cathode assembly are the beam voltage, beam current, and beam diameter at the beam waist point in the klystron. These in turn help to determine the current density at the cathode for a desired lifetime as shown above. Preliminary calculations have been made using these four parameters to define a suitable Pierce Gun [23]. In these calculations, the cathode spherical diameter was compensated for spherical aberration effects [24], and particular attention was paid to finding an optimised beam convergence angle. Table 2 gives a first estimate of the parameters of an individual CLIC MBK gun-cathode structure resulting from these calculations.

TABLE 2. CLIC MBK's single cathode preliminary parameters

\begin{tabular}{lcc}
\hline Parameter & Value & Unit \\
\hline Beam voltage & 154 & $\mathrm{kV}$ \\
Beamlet beam current & 15.04 & $\mathrm{~A}$ \\
Cathode current density & 2 & $\mathrm{~A} / \mathrm{cm}^{2}$ \\
Waist point beam radius & 0.55 & $\mathrm{~cm}$ \\
Gun perveance $\times 10^{6}$ & 0.25 & $\mathrm{~A} \times \mathrm{V}^{-3 / 2}$ \\
Beam area compression ratio & $8: 1$ & - \\
Cathode disk radius & 1.547 & $\mathrm{~cm}$ \\
Beam convergence half angle & 10.75 & degrees \\
Cathode spherical diameter & 8.28 & $\mathrm{~cm}$ \\
Beam throw - cathode vertex to beam waist & 12.5 & $\mathrm{~cm}$ \\
Maximum electric field level & 35 & $\mathrm{kV} / \mathrm{cm}$ \\
Beam pipe diameter & 2.0 & $\mathrm{~cm}$ \\
Confined flow focusing field & $600 \ldots 750$ & $\mathrm{G}$ \\
\hline
\end{tabular}




\section{MBK LAYOUT}

The overall gain (the ratio of peak output power to input drive power) will determine the minimum number of cavities that will be required. The CLIC bandwidth requirement is small (typically a few $10^{-3}$ ) so that we can assume that all gain cavities are tuned to approximately the same fundamental frequency and not stagger-tuned. A second harmonic cavity is used to improve the bunching efficiency. Commercially available solid-state RF amplifiers are available at this frequency for use as the input $\mathrm{RF}$ power source, and can reliably produce $300 \mathrm{~W}$. An overall gain of $53 \mathrm{~dB}$ is then required from the klystron RF structures to develop the desired $50 \mathrm{MW}$ peak power at the output. The klystron would then typically consist of 5 cavities: an input cavity, two gain cavities, a second harmonic cavity and an output cavity. A possible configuration for the CLIC multi-beam klystron is shown in Fig. 9.

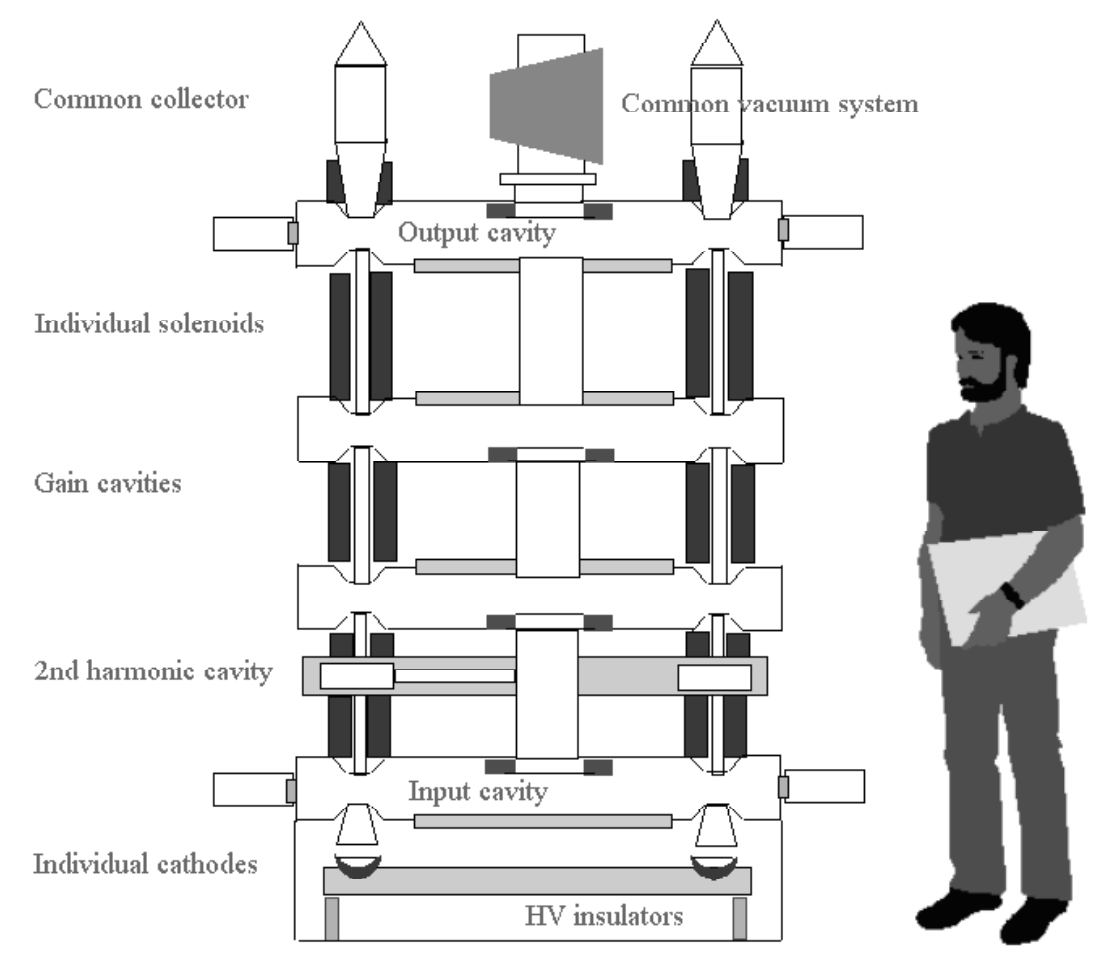

FIGURE 9: General layout of CLIC 937 MHz, 50 MW MBK.

The conceptual layout shows focusing solenoids around each beamlet. A focusing magnetic field of $600 \mathrm{G}$ to $700 \mathrm{G}$ will be needed to steer each $150 \mathrm{kV}, 15 \mathrm{~A}$ beam. A conventional solenoid will require about $1 \mathrm{~kW}$ per beamlet, and this will reduce the overall klystron efficiency. This is however just one approach - an alternative is to use PPM focusing methods, similar to those proposed for the SLAC 1.5 GHz MBK [16].

Figure 9 indicates a common collector. One could consider individual collectors with a common cooling water supply; this seems however more expensive to manufacture. The important design parameters for the collector are the mean and peak power to be dissipated, and the surface area on which the electron beam impinges. The 
minimum amount of cooling water (turbulent flow) required is generally estimated as 3 liters/minute for each kilowatt of average RF power dissipated.

\section{ACKNOWLEDGEMENTS}

The authors would like to acknowledge I. Wilson for his enthusiastic support and encouragement as well as for many useful discussions and comments. P. Pearce contributed the cathode specifications.

\section{REFERENCES}

1. The CLIC Study Team, "A 3 TeV e+e- Linear Collider based on CLIC Technology," CERN 2000-008, 2000.

2. P. Pearce, "The L-band klystron-modulator RF power system for CLIC", in IEE Pulsed Power 2000 Symposium, Digest No 00/053, London, 2000.

3. E. Gelvich, et al., "The new generation of high power MBK," IEEE Trans. Microwave Theory Tech. 41(1), 1519 (1993).

4. V. E. Balakin and I. V. Syrachev, "VLEPP RF Power Multiplier," in Proc. $3^{\text {rd }}$ Int. Workshop on Next Generation Linear Collider, Branch INP, Protvino, Russia, 1991.

5. I. Syratchev, "RF pulse Compressors systems for CTF3," in $5^{\text {th }}$ MDK Workshop, CERN, Geneva, 2001.

6. T. G. Mihran, "The effect of drift length, beam radius and perveance on klystron power conversion efficiency," IEEE Trans. Electron Dev. 14(4), 201-206 (1967).

7. R. R. Warnecke and P. R. Guenard, Tubes A Modulation Vitesse, Paris:Gauthier-Villars, 1951.

8. R. S. Symons, "Scaling laws and power limits for klystrons," in IEDM, 1986.

9. R. B. Palmer, et al., "An immersed field cluster klystron,” SLAC-PUB-5026, 1989.

10. P. B. Wilson, "RF power sources for 5-15 TeV linear colliders," in Int. Workshop on Pulsed RF Sources for Linear Colliders, Shonan Village, Japan, April 1996.

11. L. Harwood, et al., "JLAB high efficiency klystron baseline design for $12 \mathrm{GeV}$ upgrade," PAC 2003, Portland, OR, May 12-16, 2003.

12. A. Sandalov, V. Pikunov, and V. Rodyakin "High efficiency conventional and relativistic klystrons," in Int. Workshop on Pulsed RF Sources for Linear Colliders, Shonan Village, Japan, April 1996.

13. Z. D. Farkas and P. B. Wilson, "Dynamic of an electron in an RF gap," SLAC-PUB-4898Rev, 1989.

14. G. Caryotakis, "A sheet beam klystron paper design," in 5th MDK Workshop, CERN, Geneva, 2001.

15. E. Wright, et al., "Development of a $10 \mathrm{MW}$, L-band multiple-beam klystron for TESLA," in PAC 2003 , Portland, OR, May 12-16, 2003.

16. G. Caryotakis, et al., "Gigawatt multibeam klystron (GMBK)," in 11th Int. Conf. on High Power Particle Beams, Prague, Czech Republic, June 10-14, 1996.

17. L. Song, et al., "Development of a 50 MW Multi-Beam Klystron at 11.424 GHz," in PAC 2003, Portland, OR, May 12-16, 2003.

18. A. Larionov, V. Teryaev, S. Matsumoto, and Y. H. Chin: "Design of multi-beam klystron in X-band," KEKPREPRINT-2002-68, 2002.

19. I. V. Syrachev, V. F. Vogel, H. Mizuno, J. Odajiri, Y. Otake, and S. Tokumoto, "The Results of RF High Power Tests of X-Band Open Cavity RF Pulse Compression System,” in Linac 1994, Tsukuba, Japan, 1994.

20. P. Brown, private communication.

21. W. R. Fowkes, et al., "Recent advances in high power windows at X-band," in High Density Microwaves, AIP Conf. Proc. 474, Pajaro Dunes, CA, 1998.

22. S. Yu. Kazakov, "High Power Testing Results of the X-Band Mixed-Mode RF Windows for Linear Colliders," in Linac 2000, Monterey, CA, 2000.

23. J. R. Pierce, Theory and Design of Electron Beams, New York:Van Nostrand, 1954, pp. 182-192.

24. R. D. Frost and O.T. Purl, "Research on electron guns for intermediate and high power traveling wave tubes," Watkins-Johnson Co., ASD rep. ASD-TR-61-635, 1962. 